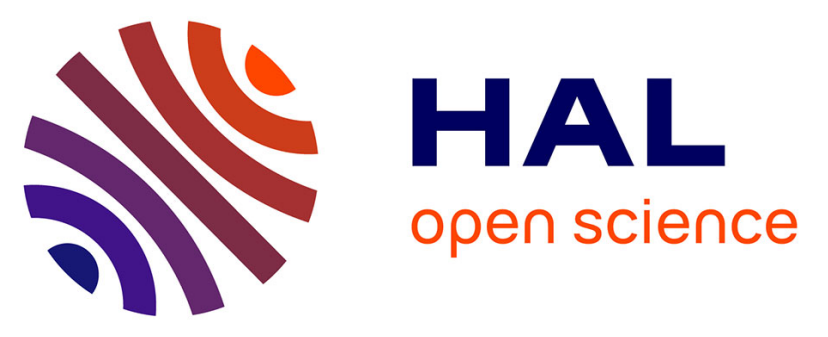

\title{
En quoi la pandémie interroge-telle les liens entre éducation, santé et sociétés?
}

J-C Verheye, F Arboix-Cala, G Jablonski-Sideris, J Descarpentries, L Bergugnat, M-H Jacques, J Guiet-Silvain, S Roy, E Darlington, J Masson, et al.

\section{To cite this version:}

J-C Verheye, F Arboix-Cala, G Jablonski-Sideris, J Descarpentries, L Bergugnat, et al.. En quoi la pandémie interroge-telle les liens entre éducation, santé et sociétés?. Recherches \& éducations, 2020, Quelle éducation avec la Covid-19, Hors série, 10.4000/rechercheseducations.9803 . hal-03480151

\section{HAL Id: hal-03480151 \\ https://cnrs.hal.science/hal-03480151}

Submitted on 24 Jan 2022

HAL is a multi-disciplinary open access archive for the deposit and dissemination of scientific research documents, whether they are published or not. The documents may come from teaching and research institutions in France or abroad, or from public or private research centers.
L'archive ouverte pluridisciplinaire HAL, est destinée au dépôt et à la diffusion de documents scientifiques de niveau recherche, publiés ou non, émanant des établissements d'enseignement et de recherche français ou étrangers, des laboratoires publics ou privés. 


\section{Recherches \& éducations}

HS | Juillet 2020

Quelle éducation avec la Covid-19

\section{En quoi la pandémie interroge-telle les liens entre éducation, santé et sociétés?}

Jean-Charles Verheye, France Arboix-Calas, Georges Jablonski-Sideris, Jacqueline Descarpentries , Laurence Bergugnat, Marie-Hélène Jacques, Jeanne Guiet-Silvain, Sylvain Roy, Emily Darlington, Julien Masson, Hélène Hagege, François Potdevin, Antoine Agraz, Eliane Rothier-Bautzer, David Authier, Jérôme Foucaud et Maryvette Balcou-Debussche

\section{(2) OpenEdition}

Édition électronique

URL : https://journals.openedition.org/rechercheseducations/9803

DOI : 10.4000/rechercheseducations.9803

ISSN : $1760-7760$

Éditeur

Société Binet Simon

Ce document vous est offert par Centre national de la recherche scientifique (CNRS)

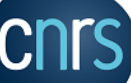

Référence électronique

Jean-Charles Verheye, France Arboix-Calas, Georges Jablonski-Sideris, Jacqueline Descarpentries ,

Laurence Bergugnat, Marie-Hélène Jacques, Jeanne Guiet-Silvain, Sylvain Roy, Emily Darlington, Julien Masson, Hélène Hagege, François Potdevin, Antoine Agraz, Eliane Rothier-Bautzer, David Authier,

Jérôme Foucaud et Maryvette Balcou-Debussche, «En quoi la pandémie interroge-telle les liens entre éducation, santé et sociétés ? ", Recherches \& éducations [En ligne], HS | Juillet 2020, mis en ligne le, consulté le 24 janvier 2022. URL : http://journals.openedition.org/rechercheseducations/9803 ; DOI : https://doi.org/10.4000/rechercheseducations.9803

Ce document a été généré automatiquement le 6 juillet 2021.

Propriété intellectuelle 


\title{
En quoi la pandémie interroge-telle les liens entre éducation, santé et sociétés?
}

\author{
Jean-Charles Verheye, France Arboix-Calas, Georges Jablonski-Sideris, \\ Jacqueline Descarpentries , Laurence Bergugnat, Marie-Hélène Jacques, \\ Jeanne Guiet-Silvain, Sylvain Roy, Emily Darlington, Julien Masson, Hélène \\ Hagege, François Potdevin, Antoine Agraz, Eliane Rothier-Bautzer, David \\ Authier, Jérôme Foucaud et Maryvette Balcou-Debussche
}

\section{Introduction}

De par sa survenue brutale, l'épidémie à Covid-19 a entrainé des bouleversements dans de nombreuses sphères de nos sociétés occidentales: fragilisation des structures de soins, déstabilisation des organisations scolaires, dérèglement de certains fonctionnements économiques et sociaux. Alors que la crise est toujours opérante, nombreux sont ceux qui envisagent déjà ses conséquences, tant cette situation semble fragiliser certains de nos fondements de pensée ou d'action.

C'est dans ce contexte que plusieurs membres de la commission scientifique d'UNIRéS, (réseau national de formateurs et de chercheurs menant des travaux sur l'éducation à la santé), se sont réunis pour croiser leurs analyses de cette situation planétaire, globale et multidimensionnelle. A travers un texte rédigé dans et sur la crise, le fruit de ce travail propose une lecture en quasi instantané de la pensée de chercheurs dont les champs d'étude sont mis en résonnance par la Covid-19. Ce texte au service de la diversité s'empare de la difficulté de prendre de la distance en pleine actualité pour lui donner du sens et en faire un événement quasi fondateur d'une réflexion collective.

L'épidémie de la Covid-19 est replacée dans une perspective historique et anthropologique avant d'envisager le questionnement sous l'angle de l'engagement communautaire, puis de l'expérience professionnelle et de la rupture biographique personnelle. Cette mise en mouvement des êtres se nourrit aussi des apprentissages qui pourraient en découler, tant sur l'esprit critique de chacun.e que sur l'enseignement 
dans diverses formes, sans oublier son accessibilité au plus fragiles. La mise en perspective des points de vue envisage cette crise comme une opportunité à réfléchir différemment et à revisiter nos champs disciplinaires dans leur dimension épistémologique pour préparer l'irréversible «après » et en être les acteurs à travers des travaux et actions à venir.

\section{Méthode}

2 La rédaction de ce texte a été conduite selon une approche basée sur l'écriture par organisation horizontale. Chaque auteur a collaboré à une partie de la rédaction à partir d'une question de départ commune, support de coopération formulé collectivement dans une logique de création d'une solidarité intellectuelle. Un membre du groupe a compilé les différentes contributions réalisées librement (Lowry, Curtis et Lowry, 2004) autour d'un plan validé collégialement. Dans un esprit de responsabilité collective et d'équité, l'ensemble des écrits a été intégré dans le texte. Afin de faciliter la mise en lien des approches des co-auteurs, une représentation graphique des points clés a été réalisée sous forme d'une carte mentale (Buzan et Buzan, 2019). Un nuage de mots réalisé en double lecture par deux membres du groupe a été proposé à partir des termes les plus utilisés dans les différents textes.

La conduite de rédaction a été facilitée par des rencontres hebdomadaires par visioconférence, de durée limitée à une heure, ouvertes à l'ensemble des contributeurs. Tous les documents de travail ont été mis à disposition sur une plateforme numérique collaborative. Chaque co-auteur pouvait ainsi participer à la réflexion collective et amender le manuscrit en cours de rédaction, selon une organisation établie collégialement. A l'issue du processus, un relecteur non rédacteur, membre du comité scientifique, a été sollicité pour opérer un regard critique sur le texte final.

\section{Une pandémie qui interpelle}

3 Avec la Covid-19, soudain, tout s'affole! Mesures, budgets, alertes... Les médias s'emparent de la question à toute heure du jour en envahissant l'univers des pensées. L'urgence suffit à justifier l'oubli des dimensions plurielles et non linéaires des individus (Lahire, 2002), leurs cultures et leurs contextes de vie. L'Etat réduit à sa propre logique, curative, rapidement mesurable, des situations sociales fort différenciées. Pendant ce temps, les maladies chroniques poursuivent leur progression à bas bruit, altérant peu à peu les personnes et les tissus sociaux, sans que les ressources de l'éducation, de la formation et des interventions contextualisées ne soient mises en exergue (Balcou-Debussche, 2016). Les vulnérabilités, durables ou passagères, dessinent ainsi un tableau qui s'assombrit régulièrement, sur fond d'inégalités sanitaires et sociales qui se renforcent. Avec l'obésité ou le diabète, personne ne s'affole, les finances n'arrivent pas et la population est rendue responsable de ses maux : «Tant pis pour elle, entend-on, elle n'avait qu'à »! D'où l'invitation à des regards critiques et distancés, notamment dans le passé...

D'un point de vue historique, avec les épidémies de peste à Byzance et en Occident au Moyen Âge, les populations des villes se sont confinées pour échapper à la contamination par les personnes malades ou par l'air miasmique. Des lazarets sont créés pour les personnes soupçonnées d'être malades, mises en quarantaine (Sidéris 
2009, Delumeau/Lequin 1987). Avec Pasteur et Koch s'opère une « révolution » qui fait que l'on immunise les populations par la vaccination. Épistémologiquement cette politique s'inscrit bien dans la biopolitique des États. Avec le VIH/sida une politique de santé est définie avec l'association des personnes concernées (Zylberman 2013). Aujourd'hui, avec le confinement de toute la population pour prévenir la transmission de la covid-19, une politique préventive conçue à l'origine au Moyen Âge est de retour et met au défi de repenser les catégories épistémologiques de la santé.

En France, la prévention de la Covid-19 est inscrite dans la doxa de la santé publique et en particulier de l'usage social et politique des résultats de la recherche en épidémiologie qui joue un rôle déterminant dans les dispositifs de prévention et l'usage social de l'éducation dans les politiques publiques de santé. Construit à partir d'une mathématisation de la vie et d'une pasteurisation dessociétés, le modèle de confinement et de gestes barrières, en absence de tests de diagnostic, de masques et de traitement s'est imposé comme un régime de contrôle pour gouverner la vie et le vivant par une forme inédite de biopouvoir et de biopolitique par la colonisation des savoirs préventifs issue de souverainetés plurielles de la santé publique épistémologiques, méthodologiques, préventives et sanitaires- (Descarpentries, 2018). Cet empire cognitif rend invisibles les dispositifs de prévention qui ont émergé comme les gestes de protection « traditionnels » et les solidarités. Forts de ce constat, comment les praxis de l'éducation à la santé peuvent-elles être un levier de transformation sociale des modèles hégémoniques de prévention, quelles que soient les aires géographiques ou les communautés concernées?

A l'instar des crises sanitaires contemporaines, la mobilisation des personnes concernées peut contribuer à la mise en œuvre de solutions efficaces, complémentaires et transformatrices. Si elles traduisent une réaction face à l'urgence, les initiatives communautaires constituent une opportunité de faire évoluer le système de santé de manière pérenne en révélant la place du patient comme réformateur social (Defert, 1994). Parmi elles, la «Ligne $C$ » (ligne d'information, d'orientation et d'écoute) issue d'un collectif de patients et de soignants dans le contexte de la Covid-19, relève de cette logique dans laquelle les savoirs expérientiels et les compétences de l'éprouvé contribuent à une dynamique pédagogique et sociale de, par et pour les usagers (Verheye, 2020). Ces savoirs non-académiques constituent un levier du partage des savoirs utiles aux individus dans leur parcours de santé, dans une logique autodidactique, autant qu'au collectif dans une complémentarité d'offre de soins, dans une logique démocratique (Jouet, 2010).

$\mathrm{Au}$ delà des usagers, différentes catégories de professionnels sont " montées au front " pour soigner, accompagner les plus vulnérables ou pour assurer la sécurité de la Nation et la continuité de la vie économique et sociale ${ }^{1}$. S'intéresser à ces professionnels plus ou moins exposés, c'est écouter leur vécu d'expériences de travail inédites confrontées aux risques, à la souffrance et au malheur humain; c'est co-analyser ces vécus dans le rapport à soi, au travail, aux autres, au politique et au monde à venir. Comment viventils cette expérience ? Quelle est la part de l'engagement et de la représentation du rôle professionnel qu'ils jouent dans la société ? Quel sens donnent-ils à leur travail dans ces conditions à risques? Présentent-ils des symptômes psychophysiologiques? Quelles sont les émotions qui les traversent? Quel regard portent-t-ils sur cet État qui gère la crise ? Comment se projettent-t-ils dans l'après? Collaborer avec ces professionnels, c'est tenter d'accéder à l'intelligibilité des situations en période de catastrophe sanitaire, politique et sociale; c'est faire œuvre de mémoire et d'alerte pour les 
expériences futures à partir d'une réflexion individuelle et collective sur les déséquilibres en cours, nous invitant à trouver des issues viables (Chabot, 2014).

Les biographies sont en effet jalonnées de segments discernables et explicables (Ricoeur, 1990), au cours desquels les identités narrative et réflexive se modifient. La rupture de linéarité liée à un événement fatidique (Javeau, 2006) produit des remaniements identitaires : les activités et les repères se reconfigurent (rapport au temps et au corps, espaces familiers ou interdits), les rôles et statuts se redéfinissent, générant une transition identitaire (Jacques, 2020) qui touche tous les statuts, genres, générations ou sphères de l'existence. Selon les ressources transitionnelles (ibid.) dont bénéficie le sujet, les phases biographiques peuvent fragiliser le Soi : a contrario, cet interstice peut aussi constituer une opportunité pour consolider son identité. En phase de menace sanitaire et de restriction des opérations sociales ordinaires, qu'est-ce qui contribue à « rassembler » l'identité, voire à la solidifier, ou au contraire, qu'est-ce qui la fragmente et la fragilise?

La pandémie actuelle nous permet ainsi de repérer des réponses humaines à la fois incertaines et tragiques, situées pour certaines dans le repli sur soi, mais également par contraste, dans le développement de la solidarité, du bien-être, associés à une indispensable réflexion en éducation à la santé. La pensée humaine devient plus flexible, en adaptant de manière créative la complexité des vraies et des fausses informations, dont la pluie incessante sur nos cerveaux ne peut que noyer leur discernement. Une des craintes de l'isolement numérique est l'indispensable réflexion sur les risques qui y est associée. L'expérience individuelle et collective via les réseaux sociaux et les ressources d'Internet fait également découvrir des usages plus diversifiés, favorisant une nécessaire relation sociale (Guiet-Silvain, 2019). L'éducation à la santé nous fait changer le regard sur soi et sur les autres (Vigarello, 2020).

\section{Une crise qui n'épargne personne}

4 Depuis plusieurs décennies, les compétences psychosociales sont considérées comme un levier de promotion de la santé (WHO, 1994); en France, les politiques de Santé publique ont commencé à s'en saisir dans leurs directives officielles. Particulièrement mise au défi dans la crise actuelle, la compétence "exercer une pensée critique» permet de développer une autonomie de pensée face à la profusion d'informations parfois contradictoires et autres fake-news, sans tomber dans les pièges du dogmatisme qui nous conduirait à épouser le point de vue de celui qui crie le plus fort, ou du relativisme pour ceux qui découvrent aujourd'hui que les sciences vivent de controverse. A l'heure où la formation à la pensée critique en santé semble avoir disparu des politiques de Santé publique au profit des recommandations et des injonctions, la crise actuelle montre l'enjeu crucial d'une formation en éducation à la santé qui assume une pensée complexe (Arboix-Calas et Caussidier, 2010), nécessaire au dépassement $\mathrm{du}$ dualisme stérile, dogmatisme versus relativisme, qui confine les esprits.

Les compétences sont aussi en jeu dans les apprentissages scolaires et l'usage des nouveaux vecteurs numériques. Depuis le début de l'épidémie, la pression sur les familles augmente autant que la fatigue des enseignants qui se disent peu accompagnés par l'administration à travers son dispositif de continuité pédagogique. Quelles valeurs l'institution attribue-t-elle à l'apprentissage quand elle dresse une liste de six offres numériques tandis que les élèves sans connexion s'évaporent dans la nature? Quelle 
considération a t-elle de l'humain quand elle poursuit son programme scolaire comme si de rien n'était? Cette nécessité d'enseigner et d'apprendre pour que la société puisse continuer d'exister (Dewey, 1916/2018) nécessiterait pourtant une déviation dans le programme scolaire, les dérobades actuelles de notre monde invitant à une connexion aux choses de la vie pour résonner avec ce fragment du monde qui nous échappe (Rosa, 2016/2018). Mais l'Education nationale y est-elle prête, à l'ère de la croissance économique à tout prix et de l'accélération technique et du rythme de vie ? Le retour à l'école invite à lever le voile sur l'envers du décor, sur les traumas et la nécessité de symboliser la catastrophe, comme d'apprendre de l'événement pour éveiller les consciences, pour s'élever et s'éduquer à la croissance de l'humain.

Au-delà du numérique, la fermeture des écoles et l'exercice de la pédagogie transférée au domicile dans le contexte du confinement révèlent une possible évolution des pratiques scolaires. Les demandes en lien avec les souffrances scolaires, le harcèlement, l'agression indirecte (Roy, 2018) et les difficultés d'apprentissage se sont arrêtées le jour de l'annonce du confinement. L'école est entrée dans la maison, les enfants ont rejoint leurs camarades électroniquement, les parents sont devenus enseignants et les enseignants sont devenus formateurs de parents-enseignants. Pour certains jeunes, la réorganisation a permis d'améliorer leur performance scolaire : ils ont travaillé à leur rythme et pris le temps de mieux comprendre ce qui leur était demandé, pour mieux y répondre. Plus apaisés, ces jeunes ont surtout la sensation qu'ils peuvent y arriver. Pour d'autres, la maittrise des normes du groupe, des règles de leurs transgressions et sanctions est simplifiée (Becker, 1963 cité par Cusson, 2016). Cette situation serait-elle une démonstration annonciatrice d'un nouveau paradigme d'organisation scolaire ? La recherche devra le documenter et tirer des enseignements de l'efficacité de certains innovations collaboratives et pédagogiques provoquées par cette crise sanitaire.

Le confinement des espaces scolaires aux foyers questionne le potentiel promoteur de santé de l'école en cette période de pandémie. Le cadre des Ecoles promotrices de santé de l'OMS des années 80 met en exergue les valeurs démocratiques dans la promotion de l'apprentissage, du développement personnel, social et de la santé de chaque élève (Darlington et al., 2019). Les actions sur le climat et l'environnement scolaire, physique mais aussi social, sont un des trois piliers pour promouvoir l'accès de tous les élèves à l'éducation et à la santé selon un principe d'équité (Langford et al., 2015), primordial et absolu. Paradoxalement, la pandémie semble tomber au plus mauvais moment dans le calendrier de la promotion de la santé à l'école. Alors que cette dernière paraissait enfin prendre la mesure de l'importance du bien-être, alors que la recherche commençait à accumuler des résultats consistants en la matière (Masson, 2019), alors que les politiques françaises commençaient à se mettre au diapason européen dans l'approche de la santé à l'école, tout s'est brusquement arrêté. La vision biomédicale et son obsession sanitaire reviennent en force, balayant toute notion de bien-être (Huebner et al., 2004). Les élèves ont été confinés chez eux, essayant, pour nombre d'entre eux, de faire tant bien que mal leur métier d'élève avec les moyens du bord. La phase de dé-confinement risque de prolonger la sentence: on désinfecte, on masque, on lave, on distancie, on interdit. Le retour à l'école pour les quelques téméraires risque d'être délétère. Comment se sentir bien dans ces conditions ? Comment apprendre dans l'ambiance oppressante généralisée ? Quelles innovations/adaptations se sont opérées pour maintenir une continuité non seulement pédagogique, mais également de lien social, favorable à la santé et au bien-être des élèves? Il y a fort à parier que cette pandémie qui contraint fortement les conduites des enfants autant que les pratiques 
des enseignants laissera des traces chez nos élèves et nous fera perdre plusieurs années pour à nouveau penser/panser une véritable école promotrice de santé.

Si la restriction de liberté générée par le confinement sollicite les capacités des individus à réguler leurs états de santé et de bien-être dans des conditions " extraordinaires ", la littératie en santé et la littératie physique (Whitehead, 2001) peuvent être des ressources pour protéger des effets néfastes du confinement ; l'enjeu consiste alors à redonner à l'individu une place centrale dans des stratégies d'éducation à la santé trop souvent réduites à la transmission de connaissances issues du monde biomédical (Potdevin et al, 2016). Le confinement a provoqué chez certains un accroissement du stress et des manifestations dépressives. La régulation des émotions a été identifiée comme une compétence psychosociale clé, d'une part parmi les facteurs de protection vis-à-vis des situations perçues comme stressantes et d'autre part pour faciliter les évolutions de comportements chez les adultes et les enfants (Cottraux, 2007). L'efficacité des techniques de méditation en termes de diminution du stress a été scientifiquement documentée (Sedlmeier et al., 2012). La régulation des émotions permet d'augmenter le pouvoir du sujet sur sa liberté intérieure (Hagège, 2018), d'autant plus primordial pour la santé mentale lorsque la liberté extérieure fait brusquement défaut. Face aux enjeux de développer les capacités individuelles des personnes, de nouvelles stratégies de promotion de la santé se sont organisées à distance, selon des formats multiples et à destination d'un large public, ce qui invite désormais à analyser leurs effets réels ou fictionnels pour prévenir les effets délétères de crises comme celles de la Covid-19.

Le caractère "extraordinaire » de ce que nous vivons met également l'accent sur les inégalités sociales. Les personnes vivant avec un handicap font partie des oubliés de la pandémie $^{2}$ et la question de l'accessibilité à la compréhension des mesures préventives liées à la pandémie est véritablement posée (Agraz, 2020). Confronté à des problèmes de mémorisation à court terme et de dépendance affective vis-à-vis des professionnels de l'école ou d'instituts spécialisés (Courbois et Facon, 2014), le public concerné par un handicap mental est particulièrement vulnérable au regard du contexte actuel. Ces deux aspects (mémorisation et dépendance) constituent des obstacles majeurs pour agir dans une vie quotidienne largement reconfigurée à l'aune du respect des gestes barrières. Les médiations éducatives procédant de la mise en place des gestes préventifs créent de nouvelles interactions sociales entre éducateurs et éduqués. A l'articulation entre éducation, santé et sociétés, la dimension d'accessibilité pédagogique peut représenter un levier d'émancipation socio-cognitive en matière d'éducation à la santé de ce public fragile.

La Covid-19 n'est pas sans révéler aussi la fragilité des systèmes. Au début de la pandémie, les regards se sont tournés vers les institutions hospitalières mises à mal par des incertitudes d'ordre clinique, matériel, organisationnel et technique. Pourtant, les soins sont constitués de configurations (Elias, 1991) dont l'hôpital n'est qu'un maillon : ville, domicile et secteur médico-social participent en mobilisant des chaînes d'interdépendances, reliées en effets domino. De cette écologie, proviennent performances ou échecs des soins (Rothier Bautzer, 2018). Les effets délétères de la crise sur plusieurs Etablissements d'Hébergements pour Personnes Agées Dépendantes (EPHAD) ont été relevés, mais on a peu interrogé les interrelations entre territoires professionnels comme au sein des territoires eux-mêmes : médecine de ville, médecine hospitalière, activités paramédicales, sociales et médico-sociales, services d'aide à la personne. Le projecteur, fixé sur la situation hospitalière, balaie trop peu ces scènes qui 
constituent le soin comme situations interconnectées, déployées dans la durée ou sur un temps plus court.

En mettant en lumière les vulnérabilités de notre époque, la pandémie interroge les représentations sociales que nous avons de l'humanité (Authier et Berger, 2015), de l'Homme et de sa position dans l'écosystème, mais aussi du pouvoir attribué à la "science ». A un niveau macroscopique, l'humain semble se percevoir au sommet de la pyramide, seul être doué de conscience, ayant éliminé tous ses prédateurs et exploitant les ressources au seul profit d'une minorité. De la même façon, la science semble encore perçue comme toute puissante alors que sa seule certitude est précisément de ne pas en avoir. Cependant, si nous parvenons à mettre en réserve quelques-unes de nos représentations, la science peut nous laisser entrevoir l'extraordinaire intrication du vivant et du non vivant au sein d'un écosystème où l'effet domino est central (Lancelot, Goldstein et Coutard, 2020). La neurobiologie montre que la conscience est présente chez de nombreux animaux (Dehaene, 2014).. Il reste quelques prédateurs non négligeables pour l'Homme même si leur taille est de l'ordre de 125 nanomètres (taille $\mathrm{du}$ SARS-CoV-2) et de nombreuses hypothèses relient les menaces faites à la biodiversité et les pandémies majeures. Celle que nous subissons actuellement peut être l'opportunité de corriger l'erreur métacognitive sociale d'une humanité toute puissante à réhumaniser face au mésusage de la conscience qu'elle perçoit de son intelligence.

\section{Conclusion}

Par sa soudaineté, son ampleur et ses répercussions, la pandémie de la Covid-19 se pose comme un nouveau révélateur des inégalités sociales de santé et de la fragilité des organisations des dispositifs de soin et de prévention, ce qui questionne nos pratiques d'enseignement et de recherche en éducation à la santé.

A l'heure où la politique de santé publique, en France, fait le choix de dissoudre les réalités individuelles au profit de la normation des comportements, au sens foucaldien lié à une gestion hégémonique du pouvoir, notamment à travers la limitation des libertés et des discours centrés sur la peur, l'heure est venue d'ouvrir de nouvelles pistes de recherche. Dans la lignée des travaux menés par notre collectif depuis plusieurs années, les éducations et des formations en santé qui ne se construisent pas dans une opposition des épistémologies sont d'entière actualité. L'expérience individuelle et collective de la pandémie de la Covid-19 a mis en réflexivité critique nos travaux, ce qui ouvre un espace d'analyse de la recherche académique dans le champ de l'éducation à la santé et de ses pratiques opérationnelles. L'éducation à la santé post Covid-19, ne pourra se suffire à l'apprentissage des gestes barrières et à la soumission volontaire ou involontaire à des mesures de protection lors de prochaines épidémies : elle a à construire, en se nourrissant d'autres apports épistémologiques et pragmatiques de nouvelles praxis de recherche et d'intervention. La crise vécue avec la Covid-19 nous donne ainsi l'opportunité de (re)penser les moyens d'agir sur la santé individuelle et collective, au profit de l'éducation à la santé de demain, en faisant appel à la solidarité et à la coopération avec les populations. 


\section{BIBLIOGRAPHIE}

Agraz, A. (2020). La philosophie du temps chez Gaston Bachelard : vers de nouveaux schèmes d'évaluation des stages pour une plus grande accessibilité pédagogique des jeunes d'IME avec un handicap mental. Recherche et Formation.

Arboix-Calas, F. et Caussidier, C. (2010). Former à l'éducation pour la santé dans la pensée complexe. In D. Berger et C. Simar (Dir.), Éducation à la santé dans et hors l'École : recherches et formations, pp 427-437.

Authier, D. et Berger, D. (2015). Recherche, analyse de conceptions chez des patients diabétiques et leurs soignants. Education Thérapeutique du Patient, 7(2), 20105.

Balcou-Debussche, M. (2016). De l'éducation thérapeutique du patient à la littératie en santé. Problématisation socio-anthropologique d'objets didactiques contextualisés. Paris : Editions des Archives Contemporaines.

Bergugnat, L. (2019). Les risques psychosociaux. In A. Vandevelde-Rougale et P. Fugier, La sociologie clinique de A à Z. Toulouse : Eres.

Buzan T. \& Buzan B. (2019). Mind map : dessine-moi l'intelligence. Paris : Eyrolles.

Chabot, P. (2013). Global burn-out. Paris : PUF.

Cottraux, J. (Eds.). (2007). Thérapie cognitive et émotions La troisième vague. Masson.

Courbois, Y. et Facon, B. (2014). Les savoirs de la psychologie cognitive. In C. Gardou et al., Handicap, une encyclopédie des savoirs. Des obscurantismes à de Nouvelles Lumières. Toulouse. Cusson, M. (2016). La criminologie. Hachette collection, 6ème édition.

De Sousa Santos, B. (2018). The end of cognitive empire. Duck University Press.

Darlington, E. et Masson, J. (2019). Articuler inégalités sociales, équité, citoyenneté et solidarités en promotion de la santé : enjeu et perspectives, Education, Santé, Sociétés, 6(1).

Defert, D. et Martel, F. (1994). Le malade du sida est un réformateur social : entretien avec Daniel Defert. Esprit, 7, 203.

Dehaene, S. (2014). Le code de la conscience. Paris : Odile Jacob.

Delumeau J. et Lequin, Y. (dir.) (1987). Les malheurs des temps. Histoire des fléaux et des calamités en France. Paris : Larousse.

Descarpentries, J. (2019). L'éducation pour la santé durable comme levier de transformation sociale. Educations, 1.

Dodier, N. (2003). Leçons politiques de l'épidémie de sida. Paris : Editions de l'Ecole de Hautes Etudes en Sciences Sociales.

Elias, N. (1991). La société des individus. Editions Fayard.

Guiet-Silvain J. (2019). L'impact des réseaux sociaux sur les relations adolescentes. Congrès de l'AREF, Bordeaux.

Hagège, H. (2018). Pour une éducation à la responsabilité. International Science and Technology Editions. 
Huebner, E.S., Suldo, S.M., Smith, L.C. \& McKnight, C.G. (2004). Life satisfaction in children and youth : Empirical foundations and implications for school psychologists. Psychology in the Schools, 41, 81-93.

Jacques, M-H. (2020). Les transitions identitaires dans les parcours d'éducation. Rennes : Presses Universitaires.

Javeau, C. (2006). Routines quotidiennes et moments fatidiques. Cahiers internationaux de sociologie 121, 227-238.

Jouet, E., Flora, L. et Las Vergnas O. (2010). Construction et reconnaissance des savoirs expérientiels des patients : note de synthèse. Pratiques de formation - Analyses. Université Paris 8.

Lahire, B. (2002). Portraits sociologiques. Dispositions et variations individuelles. Paris : Nathan.

Lancelot, R., Goldstein, T. et Coutard, B. (2020). Coronavirus. Et maintenant ? Sciences \& Vie, 1232, 62-83.

Langford, R., Bonell, C., Jones, H., Pouliou, T., Murphy, S., Waters, E., ... et Campbell, R. (2015). The World Health Organization's Health Promoting Schools framework : a Cochrane systematic review and meta-analysis. BMC Public Health, 15(1), 130.

Lowry PB. Curtis A. Lowry MR. (2004). Building a Taxonomy and Nomenclature of Collaborative Writing to Improve Interdisciplinary Research and Practice. The Journal of Business Communication, 41, 66-99.

Masson, J. (2019). Bienveillance et réussite scolaire. Paris : Dunod.

Potdevin F, Porrovecchio A, Dieu O, Racodon M, Schnitzler C. (2017). Éduquer à la santé par l'activité physique : quelles connaissances et quels modèles de santé en EPS ?Éducation, Santé, Sociétés, 3(2), 137-152.

Ricoeur, P. (1990). Soi-même comme un autre. Paris : Seuil.

Rosa, H. (2018). Résonance, une sociologie de la relation au monde. Paris : La Découverte.

Rothier Bautzer, E. (2018). L'écologie des groupes professionnels. Education, Santé, Sociétés, 4(2), 101-113.

Roy, S. (2018). Prévenir l'agression indirecte ; expérimentation et adaptation d'un programme de prévention en collège - Thèse. Université de Lyon.

Sedlmeier, P., Eberth, J., Schwarz, M., Zimmermann, D., Haarig, F., Jaeger, S., \& Kunze, S. (2012). The psychological effects of meditation : A meta-analysis. Psychological Bulletin, 138(6): 1139-1171.

Sidéris, G. (2009). Quelques aspects des catastrophes naturelles à Byzance et de leurs effets sur la société. In L. Buchet, C. Rigeade, I. Séguy et M. Signoli. Vers une anthropologie des catastrophes. Antibes/Paris, APDCA/INED, pp. 163-178.

Verheye, J-C. (2019). L'intervention des patients en éducation thérapeutique : entre optimisation des apprentissages et évolution du système de soins. Education, Santé et Sociétés (6)1, 205-224.

Vigarello, G. (2020). La santé passe désormais avant toute autre chose. Le Un, 294.

Whitehead, M. (2001). The Concept of Physical Literacy. European Journal of Physical Education, 6(2), 127-138.

World Health Organization. (1994). Life skills education for children and adolescents in schools. Geneva : World Health Organization.

Zylberman, P. (2013). Tempêtes microbiennes. Paris : Gallimard. 


\section{NOTES}

1. Ordonnance $\mathrm{n}^{\circ} 2020-323$ du 25 mars 2020 portant mesures d'urgence en matière de congés payés, de durée du travail et de jours de repos.

2. Le Journal du Dimanche, 04.04.20

\section{RÉSUMÉS}

Durant l'épidémie à Covid-19, des membres de la commission scientifique d'UNIRéS ont croisé leurs analyses de la crise sanitaire. En associant une écriture par organisation horizontale et une carte mentale, ils ont produit un texte collectif qui éclaire la situation sous l'angle de l'histoire, de l'anthropologie, de l'engagement communautaire, de l'expérience professionnelle et de la rupture biographique personnelle. Leurs réflexions mettent l'accent sur la crise épistémologique et portent sur les apprentissages, l'esprit critique de chacun et l'enseignement dans son approche, ses formes et son accessibilité aux plus fragiles. Cette production plurielle donne l'opportunité de (re)penser les moyens d'agir sur la santé individuelle et collective, au profit de l'éducation à la santé de demain, en faisant appel au collectif, à la solidarité et à la coopération avec les populations.

During the Covid-19 epidemic, members of the UNIRéS network's scientific committee crossed their analysis of the health crisis. By combining writing by "horizontal organization" with a concept map, they elaborated a collective text, which enlightens the current situation from the perspectives of history, anthropology, community involvement, professional experience and personal biographical breakdown. Their reflexions emphasize the epistemological crisis and relate to the learnings from the pandemic and critical understandings of it, as well as teaching with regards to its approach, its forms and its accessibility to the most vulnerable. This plural production is an opportunity to (re) think the means of action on individual and collective health, for the benefit of tomorrow's health education, with a view of appealing to the collective and also to solidarity and cooperation with populations.

\section{INDEX}

Mots-clés : covid-19, éducation à la santé, santé publique, recherche, pratiques, épistémologies, expérience, engagement, apprentissage

Keywords : covid-19, health education, public health, research, pratices, epistemologies, experience, commitment, learning

\section{AUTEURS}

\section{JEAN-CHARLES VERHEYE}

Université Sorbonne Paris Nord, laboratoire Éducations et Pratiques de Santé (UR 3412)

Commission Scientifique UNIRéS : www.unires-edusante.fr 


\section{FRANCE ARBOIX-CALAS}

Université de Montpellier, laboratoire LIRDEF (Laboratoire Interdisciplinaire de Recherche en Didactique, Education et Formation)

Commission Scientifique UNIRéS : www.unires-edusante.fr

\section{GEORGES JABLONSKI-SIDERIS}

Sorbonne Université (INSPE). Equipe Monde Byzantin (UMR 8167) au Collège de France Commission Scientifique UNIRéS : www.unires-edusante.fr

\section{JACQUELINE DESCARPENTRIES}

Université de Paris 8-Vincennes, Ecole Doctorale de Sciences Sociales, laboratoire EXPERICE Commission Scientifique UNIRéS : www.unires-edusante.fr

\section{LAURENCE BERGUGNAT}

Université de Bordeaux, laboratoire Cultures Education Sociétés, INSPE Aquitaine Commission Scientifique UNIRéS : www.unires-edusante.fr

\section{MARIE-HÉLÈNE JACQUES}

Université de Limoges, Unité de Recherche FrED (Education et Diversité en espaces francophones)

Commission Scientifique UNIRéS : www.unires-edusante.fr

\section{JEANNE GUIET-SILVAIN}

Université de Paris, CAREF, Centre amiénois de recherche en éducation et formation, Amiens Commission Scientifique UNIRéS : www.unires-edusante.fr

\section{SYLVAIN ROY}

Université Claude Bernard, Lyon 1, laboratoire HESPER (Health Services and Performance Research)

Commission Scientifique UNIRéS : www.unires-edusante.fr

\section{EMILY DARLINGTON}

Université Claude Bernard - Lyon1, laboratoire Parcours Santé Systémique (P2S) Commission Scientifique UNIRéS : www.unires-edusante.fr

\section{JULIEN MASSON}

Université Claude Bernard - Lyon1, laboratoire Parcours Santé Systémique (P2S)

Commission Scientifique UNIRéS : www.unires-edusante.fr

\section{HÉLÈNE HAGEGE}

Université de Limoges, Unité de Recherche FrED (Education et Diversité en espaces francophones)

Commission Scientifique UNIRéS : www.unires-edusante.fr

\section{FRANÇOIS POTDEVIN}

Univ. Lille, Univ. Artois, Univ. Littoral Côte d'Opale, ULR 7369 - URePSSS - Unité de Recherche Pluridisciplinaire Sport Santé Société Commission Scientifique UNIRéS : www.unires-edusante.fr 


\section{ANTOINE AGRAZ}

Université de Limoges, Unité de Recherche FrED (Education et Diversité en espaces

francophones)

Commission Scientifique UNIRéS : www.unires-edusante.fr

\section{ELIANE ROTHIER-BAUTZER}

Université de Paris, CERMES3, UMR 8211 CNRS-INSERM-EHESS

Commission Scientifique UNIRéS : www.unires-edusante.fr

\section{DAVID AUTHIER}

Université de Limoges, Unité de Recherche FrED (Education et Diversité en espaces francophones)

Commission Scientifique UNIRéS : www.unires-edusante.fr

\section{JÉRÔME FOUCAUD}

Université Sorbonne Paris Nord, laboratoire Éducations et Pratiques de Santé (UR 3412) Commission Scientifique UNIRéS : www.unires-edusante.fr

\section{MARYVETTE BALCOU-DEBUSSCHE}

Université-INSPé de La Réunion, Laboratoire Icare (Institut Coopératif Austral de Recherche en Education)

Commission Scientifique UNIRéS : www.unires-edusante.fr 